\title{
Modelling and Simulation of a Complete System of Energy Transmission
}

\author{
A. $\operatorname{DOUYERE}^{(1)}, \mathrm{J}^{-D}$. LAN SUN LUK
and J-P CHABRIAT
$*(1)$ \\ (1) LE2P, http://www2.univ-reunion.fr/ actes/ \\ University of La REUNION, REUNION (FRANCE) \\ alexandre.douyere@univ-reunion.fr
}

\begin{abstract}
This article presents an equivalent circuit approach used for the modelling of a system consisting of an antenna and a RF/DC rectifying circuit (rectenna) at $2.45 \mathrm{GHz}$. The measurements and simulations steps used for parameter extraction and model validation are presented. The major interest in this approach is the ability to study a complete system of energy transmission in a single simulator environment. This method is illustrated in the case of a microwave transmission without any modulation, that is to say the derived model can be easily integrated. The results obtained using this simple example show a relatively good agreement with the experiment and can form the basis for further developments of more complex systems.
\end{abstract}

\section{Introduction}

Wireless Power Transmission by microwaves at $2.45 \mathrm{GHz}$ can be used for remotely supplying power to very low power smart sensors or to transmit energy from Space Power Satellites to the Earth, [1] thus providing clean energy supply at a much wider scale. When transmitting either telecommunication signals or a cw RF beam from a transmitter to a receiver, random variations of atmospheric conditions may occur together with environmental or geometrical changes around the communicating elements. We are currently developing means to alleviate the negative effects that these random perturbations can have on the rectenna energy conversion efficiency. To design these solutions one needs to have a model of the non linear Schottky diode rectifier together with the feeding antenna. This model needs to be sufficiently precise while at the same time being simple to implement in conventional RF circuit design tools. Consequently, we have chosen to derive an equivalent circuit model of the antenna and the transmission channel following the previous work of Gerrits et al., [2]. The model parameters extracted are based on measurements made on the half wavelength dipole antenna used in the rectenna. The antenna+rectifier system can subsequently be simulated and optimized for performance using a harmonic balance simulator. The first and the second parts of this article present the equivalent circuit used to model the antenna, the transmission channel and the parameter extraction process. Simulation of the derived rectenna model is presented in the subsequent parts where the obtained results are compared with experiments and show a relatively good agreement. It is believed that this approach can provide a simple and yet efficient simulation framework to further study non stationary perturbation damping circuits. 


\section{Modelling of a Dipole Antenna}

A half wavelength type dipole antenna with a $2.45 \mathrm{GHz}$ resonating frequency will be modelled. This antenna is simple to build and simulate while allowing the fabrication of large collecting arrays which are transparent to light. The latter property is of particular concern and becomes a quality when it turns to collecting large amount of power coming from Space Solar Plants at low power densities. Indeed, large rectenna arrays are needed and must not sacrifice land usage and biological life.

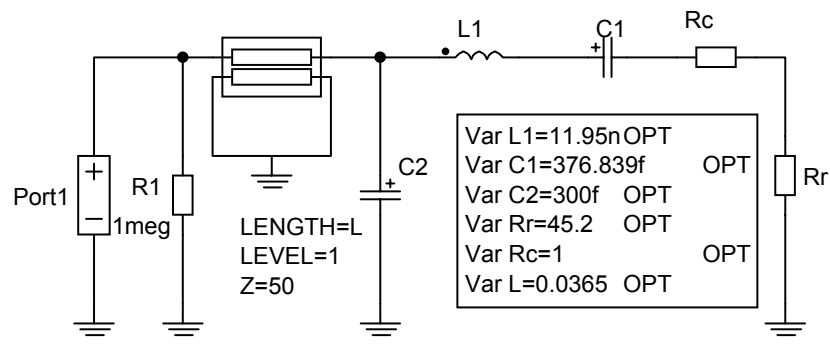

Fig. 1: Equivalent electrical circuit of a $2.45 \mathrm{GHz}$ resonant frequency half wavelength dipole antenna.

Figure 1 shows the equivalent circuit used to model the electrical behaviour of the antenna in response to an incoming RF beam. Port 1 represents the RF beam incident power source. $\mathrm{R}_{\mathrm{C}}, \mathrm{R}_{\mathrm{r}}, \mathrm{L}_{1}$ et $\mathrm{C}_{1}$ are electrical elements whose value influences the resonating frequency and quality factor Q. Elements TLINE and C2 are included here to allow for the adjustment of the equivalent electrical length. Power dissipated in $R_{C}$ corresponds to the conduction losses whereas resistance $\mathrm{R}_{\mathrm{r}}$ corresponds to the radiating losses. This model will subsequently be studied in a frequency band from $1 \mathrm{GHz}$ to $3 \mathrm{GHz}$ encompassing the resonating frequency of the dipole used and corresponding to the measuring equipment at hand at the time of writing this paper.

\section{Equivalent Circuit Parameter Estimation}

A 'Gradient' method used in the APLAC software, [3] aims at minimizing a function representing the error from the simulated $\mathrm{S}_{11}$ parameter of the dipole to the measured one in the chosen frequency band. The $S_{11}$ parameter measurement is performed using a vector network analyzer. The reference plane is determined from the calibration using $3.5 \mathrm{~mm}$ standards. On figure 2 and 3 the measured $\mathrm{S}_{11}$ parameters are compared with the ones simulated after optimization. The corresponding values of the equivalent circuit elements are the ones displayed in figure 1.

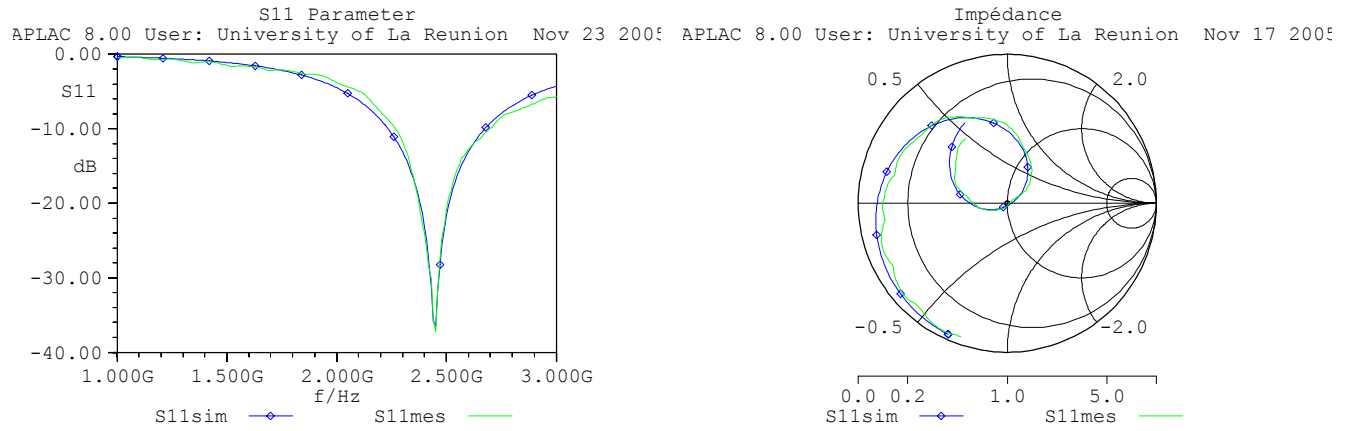

Fig. 2: Simulated and measured $\mathrm{S}_{11}$ magnitude Fig. 3: Smith chart representation of optimized curves compared after optimization. and measured $\mathrm{S}_{11}$ parameter. 
Because of this relatively good agreement the equivalent circuit is expected to provide a correct model of the circuit behaviour in the frequency band of interest. Moreover, with the reciprocity principle for an antenna, it should be possible to use the derived antenna model for both the reception and transmission of RF signals.

\section{RF Transmission Model Validation}

In order to validate the model of this antenna that we obtained in simulation, an experimental test setup was developed to measure the energy transmission between two identical $\lambda / 2$ dipoles at various distances. Similarly, simulations were performed where an equivalent model circuit of the transmitting and receiving antenna was used, [2]. Path loss is accounted for by using a controlled source $\mathrm{E}_{1}$. The gain of the source is frequency independent but is a function of the distance $\mathrm{x}: G_{\text {ain }}=-0.004 * x+0.133$. The propagation delay is accounted for by a transmission line of appropriate length. Figure 4 and 5 show the comparison between the simulated S21-parameter and the measured one.

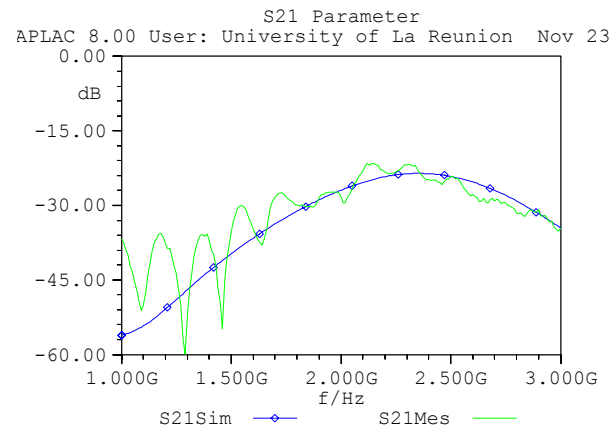

Fig. 4: Comparison between the simulated S21parameter and the measured one.

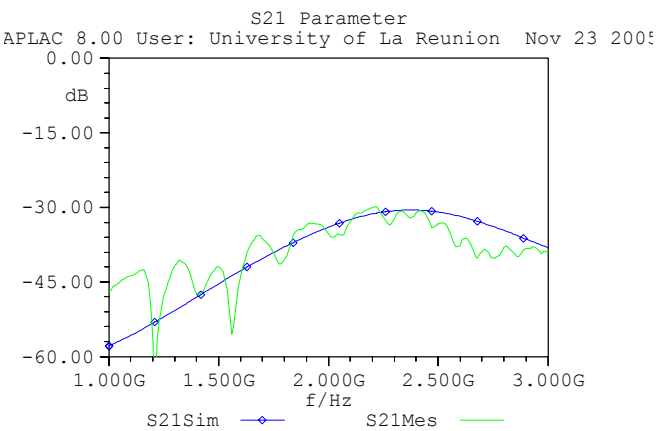

Fig. 5: Comparison between the simulated S21parameter and the measured one.

\section{Microwave Power Transmission System Modelling}

In this part, we try to demonstrate the ability of this modelling procedure to fully describe the antennas and the transmission channel behaviour. The design and test of a rectenna (rectifier + antenna) is presented thereafter (figure 6). The purpose of this study is to experimentally estimate the DC energy collected by a constant resistive load $\mathrm{R}_{\text {load }}=73 \Omega$ connected at the output of the rectenna, as a function of the transmitting distance and for a given transmitted power $\mathrm{P}=26 \mathrm{dBm}$ and to compare this data with the simulations of the same system using the equivalent circuit model approach developed in this paper. For practical reasons, this study was realized for distances ranging from $10 \mathrm{~cm}$ to $30 \mathrm{~cm}$. Figure 7 shows the measured and simulated results obtained during this study. 


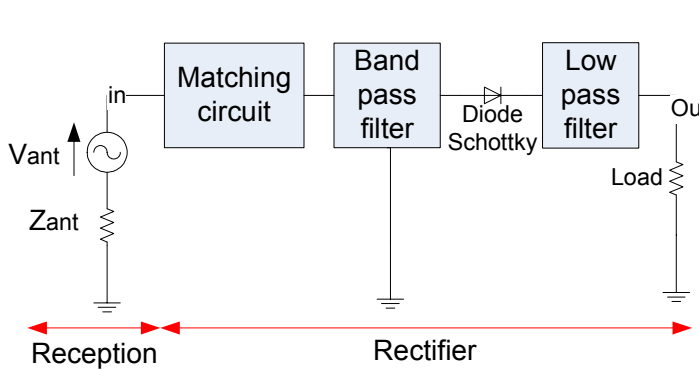

Fig. 6: Structure of the Rectenna.

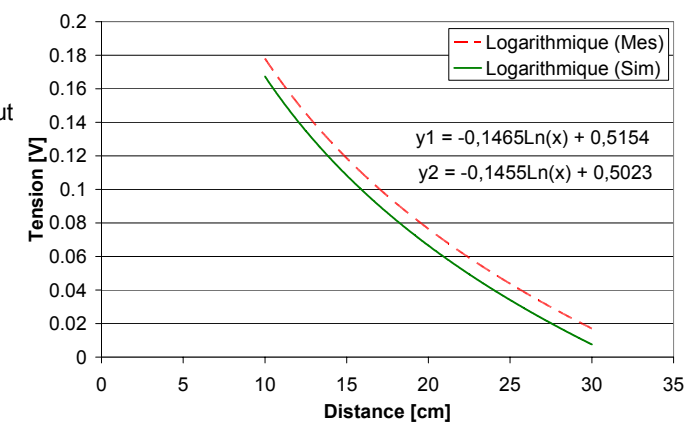

Fig. 7: DC voltage at rectenna output as a function of transmission distance.

It can be noticed that a very good agreement is obtained. The observed offset stays within the margin of error due to fabrication tolerances and measurement errors. Dependence with the distance is very well predicted by the simulator and this shows that the behaviour of the rectifier seems to be correctly taken into account by the equivalent circuit model used in this work.

\section{Conclusion}

We have presented an equivalent circuit model of a complete Wireless Power Transmission system. Models of each element of the system can be identified using experimental measurement results. The major interest in this approach is the ability to fully optimize the circuit in a single simulator environment. For example, using this equivalent circuit model, the complete system of energy transmission could be optimized for low spurious emissions and high rectenna efficiency. In a future work, impedance of the equivalent circuit representing the antenna may be optimized and the approach will require us to synthesize the antenna exhibiting the same model. This may require the modelling of more complex antenna structures.

\section{References:}

[1] SUSUMU Sasaki and YOSHIHIRO Naruo (1999):

"Guidelines for Development of SPS 2000 Educational Model", (ISAS), 3-1-1 Yoshinodai, Sagamihara, Kanagawa 229-8510, JAPAN.

[2] GERRITS John, HUTTER Andreas, AYADI Jaouhar, FARSEROTU John (2003):

"Modelling and simulation of a dipole antenna for UWB applications using equivalent SPICE circuit", International Workshop on Ultra Wideband Systems (IWUWBS), June 2003, Oulu, Finland.

[3] http://www.aplac.com 